\title{
Ciência política e análise de redes: uma metodologia para o mapeamento de comunidades temáticas
}

\author{
Political science and network analysis: a methodology for the mapping of thematic communities
}

\author{
Vandro Elaino Feretti ${ }^{1}$ \\ Ivan Jairo Junckes ${ }^{2}$ \\ Augusto Junior Clemente ${ }^{3}$
}

\begin{abstract}
Resumo
O presente artigo traz os resultados da elaboração de uma metodologia para o mapeamento de comunidades temáticas em artigos publicados em língua inglesa em uma determinada área de conhecimento. A questão que orientou os trabalhos foi: quais os temas e autores centrais que publicam em língua inglesa na área de ciência política abordando a análise de redes sociais? A coleta inicial de metadados foi realizada no portal Google Acadêmico e resultou no corpus de 204 artigos publicados entre 2011 e 2016 em 20 periódicos que apresentam índice "h5". Os artigos completos foram dispostos em planilha de dados para geração de uma rede de autores e palavraschave submetida para análise e geração de grafos no software de análise de redes sociais Gephi. Os resultados permitiram identificar dezoito comunidades temáticas em vinte periódicos internacionais, todos com classificação A1 no Qualis Periódicos. A metodologia utilizada provou-se generalizável para qualquer campo temático, de formas a proporcionar aos pesquisadores uma exploração facilitada da produção acadêmica em língua inglesa.

Palavras-chave: Ciência Política. Análise de Redes Sociais. Análise Semântica.
\end{abstract}

\begin{abstract}
The present article presents the results of the elaboration of a methodology for the mapping of thematic communities in articles published in English in a certain area of knowledge. The question that guided the work was: what are the central themes and authors that publish in the English language in the area of political science addressing the analysis of social networks? The initial collection of metadata was carried out in the Google Scholar portal and resulted in the corpus of 204 articles published between 2011 and 2016 in 20 journals that present an "h5" index. The complete articles were arranged in a spreadsheet for generating a network of authors and keywords submitted for analysis and generation of graphs in Gephi social network analysis software. The results allowed the identification of eighteen thematic communities in twenty international journals, all with A1 classification in the Periodic Qualis. The methodology used has proven to be generalizable to any subject field, in a way that gives researchers a facilitated exploration of academic production in the English language.
\end{abstract}

Keywords: Political science. Analysis of Social Networks. Semantic Analysis.

\footnotetext{
1 Mestrando no Programa de Pós-Graduação em Desenvolvimento Territorial Sustentável da Universidade Federal do Paraná (PPGDTS/UFPR). E-mail: vvandro@gmail.com

2 Doutor em Sociologia Política (UFSC). Professor do PPGDTS/UFPR. E-mail: ivanjj@ufpr.br

3Doutor em Ciência Política. Professor da UFPR e do Mestrado Profissional em Políticas Públicas da Universidade Federal do Pampa (UNIPAMPA). E-mail: augustoclemente@ufpr.br
} 


\section{Introdução}

Apresentamos neste estudo os resultados obtidos com a análise de 204 artigos da área da ciência política que abordam a análise de redes como metodologia e foram publicados nos principais periódicos anglófonos entre os anos de 2011 e 2016. Para identificar as comunidades temáticas, formadas nas respectivas publicações, utilizamos justamente a análise de redes, pois pouco sentido faria utilizarmos recursos metodológicos não relacionais para exploração de um conteúdo essencialmente relacional. Respondemos com o trabalho a seguinte questão de pesquisa: quais os temas e autores centrais que publicam em língua inglesa na área de ciência política abordando a análise de redes sociais? O conjunto de procedimentos utilizados para responder a este questionamento gerou uma ferramenta generalizável com a qual é possível "atalhar" caminhos e identificar os principais autores a serem buscados e lidos em uma determinada área do conhecimento.

A análise de redes sociais (ARS) é uma metodologia em franca expansão no mundo acadêmico. Sua trajetória está sistematizada em Berry et al. (2004), onde os autores situam o uso da teoria de redes como referencial teórico nas áreas de sociologia, ciência política e administração/gestão pública. A trajetória da ARS abrange desde os estudos sociométricos de Moreno nos anos 1930, e as modelagens matemáticas desenvolvidas pelos estruturalistas de Harvard nos anos 1960/70, até os trabalhos recentes sobre inovação e mudanças políticas, configuração de agenda pública e redes políticas. Todavia, nos debatemos com uma barreira considerável para o desenvolvimento de trabalhos na área, pois grande parte da bibliografia que utilizamos está publicada em língua inglesa.

Para vários pesquisadores iniciantes o tempo necessário para leitura e compreensão desses textos é um limite, ou mesmo um impeditivo para os estudos em ARS. Sob estas condições percebemos a oportunidade para realizarmos um trabalho que se tornasse referência de indicação daquelas leituras centrais e indispensáveis quando exploramos um determinado campo de conhecimento. Com o objetivo de suprir esta lacuna acadêmica, resolvemos investigar como a análise de redes sociais tem sido utilizada nas pesquisas realizadas em ciência política e publicadas em periódicos anglófonos.

O caminho "normal" para realizarmos a pesquisa seria a bibliometria, pois é a metodologia frequentemente utilizada para sistematizar dados de publicações, apontando obsolescências e tendências, relativas a um campo de conhecimento. Esses estudos utilizam métricas próprias para “medir", descrever e analisar os atributos (em geral títulos, autores e palavras-chave) de seus objetos-publicações. Entretanto, o conjunto das relações estabelecidas entre os autores e suas 
publicações não poderia ser explorado através da bibliometria. Precisávamos, portanto, aplicar a análise de redes para estudar o que se tem produzido em ciência política e análise de redes. Assim o fizemos e inovamos, inspirados nos estudos de Almeida, Arencibia e Yohannis (2007), Clemente (2015), Silva e Silva (2016), Silva e Pereira (2016) e Gama e Carvalho (2017).

O percurso deste trabalho é apresentado a seguir em quatro partes além desta introdução. $\mathrm{Na}$ primeira parte, apresentamos algumas discussões a respeito dos estudos desenvolvidos em bibliometria, em análise de redes sociais e relacionados a aplicabilidade da análise semântica para a pesquisa bibliométrica. Após, apresentamos a trajetória da pesquisa, os dilemas e dificuldades enfrentadas no levantamento inicial e no processamento e análise dos dados. Buscamos descrever os passos e procedimentos adotados visando cumprir com o respeito ao princípio elementar da refutabilidade acadêmica. No terceiro tópico, apresentamos os resultados da pesquisa e descrevemos minimamente as comunidades temáticas identificadas na rede de autores e palavraschave. Para a visualização dos resultados utilizamos de grafos comentados para apresentar as relações estabelecidas na rede indicada. Por último, tecemos nossas considerações, avaliando os resultados alcançados com nosso estudo e os núcleos centrais nas comunidades temáticas identificadas.

\section{Análise relacional de conteúdo: a associação metodológica da bibliometria e da análise de redes}

A bibliometria estuda os aspectos quantitativos da produção, da disseminação e do uso da informação registrada. Os estudos de natureza bibliométrica são comumente utilizados para quantificar dados oriundos de publicações referentes a um determinado tema que está sendo discutido ou a uma determinada área do conhecimento. Também para mostrar as tendências da área estudada, os estudos que se tornaram obsoletos em diversos campos da ciência, demonstrar a forma como os estudos empíricos estão sendo realizados e o interesse dos pesquisadores em determinado assunto.

O termo bibliometria se popularizou a partir de 1969, quando Alan Pritchard sugeriu que ela deveria substituir o termo bibliografia estatística. Em destaque na bibliometria está a linha de pesquisa que estuda as citações. Por meio delas é possível identificar os termos centrais e os elos entre citações em um campo científico. Ainda, pode-se inferir, por exemplo, autores mais citados e trabalhos mais reconhecidos de periódicos que compõem um campo científico determinado, sua aplicabilidade ou obsolescência na literatura, etc. Conforme Ferreira (2010, p. 05) a bibliometria em 
especial é utilizada para obter indicadores de produção científica. É uma métrica que afere o tamanho da elite de determinada população de autores a partir de citações a despeito de um mesmo autor em um campo definido.

A análise bibliométrica é um método que pode identificar tendências de pesquisa e questões com base em informações de publicações históricas (BUNEA; BAUMGARTNER, 2014). Quando feitas em periódicos digitais são costumeiramente realizadas a partir do título, autor e palavras-chave (LIMA, 2008). As palavras-chave utilizadas pelos autores nos artigos são também habitualmente utilizadas na análise bibliométrica para uma maior compreensão do status de pesquisa e tendências de desenvolvimento das temáticas identificadas (LI; HAN; LU, 2018).

Assim, a bibliometria oferece mecanismos para elencar grupos e estruturas matematicamente construídas. Apresenta resultados exatos, fixos, estáticos e mensurados conforme os termos agregados à pesquisa bibliométrica. Todavia, dois limites dificilmente são transpostos nos trabalhos bibliométricos. Primeiro, as nuances linguísticas composicionais deixam de ser identificadas e relativizadas, como por exemplo, os sinônimos, homônimos, singular, plural, parassínteses e outros que podem insurgir em resultados questionáveis. Segundo, e mais significativo, o conjunto relacional formado pelos grupos de palavras-chaves e autores não tem sido identificado pelos recursos bibliométricos correntes, especialmente as comunidades temáticas formadas pelas relações de vizinhança e proximidade apresentadas por esses relacionamentos. Tais limitações podem ser superadas com o uso da teoria dos grafos e a metodologia da análise de redes sociais, assumindo, no trabalho bibliométrico, um caráter de análise semântica.

A análise de redes sociais (ARS), também conhecida pela expressão inglesa social network analysis (SNA), toma como unidade fundamental de análise as relações entre atores sociais. Os atores podem ser indivíduos, organizações, cidades, grupos, publicações e, inclusive, termos ou palavras. O conceito de análise de redes vem sendo utilizado em muitas áreas do conhecimento; em estudos de estrutura de parentesco, mobilidade social, citações científicas, contatos entre os membros de grupos sociais, estrutura de classe, análise de financiamento de eleições, estudos de grupos religiosos, entre outros. Em estudo sobre a análise de redes nas ciências sociais, Borgatti et al (2009, p. 892) afirmam que "Para cientistas sociais a teoria de redes tem sido uma mina de ouro, produzindo explicações para os fenômenos sociais em uma ampla variedade de disciplinas...”.

A ARS teve suas raízes firmadas na psicologia social de Jacob Moreno, sendo denominada de Sociometria (SCOTT, 1988, p. 110). Como forma de conceituar as estruturas de pequenos grupos produzidos através da amizade e padrões de interação informal. Moreno utilizou a metáfora da rede com base em conceitos sociológicos e por meio de indicações orientou uma análise com base nas 
orientações de amizade e lançou as bases para o estudo da "dinâmica de grupo", que compreendeu a espinha dorsal da psicologia social americana nos anos 1950 e 60 (SCOTT, 1988, p. 111).

A partir das abordagens da análise da estrutura social nos últimos vinte anos, com seu caráter matemático e de representação centrada na imagem do entrelaçamento das relações sociais, a sociologia estrutural tem se firmado como campo e metodologia de estudos (MIZRUCHI, 2006). Ao buscar representar os relacionamentos entre indivíduos, Scott (1988, p. 130) esclarece que pessoas são estruturas complexas, como articulações complexas de papéis e relacionamentos. A partir deste ponto de vista, o agente humano é intrinsecamente multidimensional.

Com o tempo a análise estrutural foi se consolidando como uma forma de investigação social com suas especificidades focais. Wellman (1997), destaca as vias de socialização das normas e atributos dedicados aos componentes. As análises se dão nos relacionamentos dentro dos grupos não nos atributos internos. A preocupação da análise de redes está na relação estabelecida entre os comportamentos e como esta relação afeta todo o conjunto (clusters) que organiza a rede em sua íntegra. Para o autor a estrutura que compõe a rede pode ser dividida em sub-redes com seus componentes, assumindo que, os grupos menores podem ou não ser tratados como eixos centrais de uma rede. Assim, Wellman define uma rede como um conjunto de laços que vinculam membros do sistema social através e além de categorias sociais e grupos fechados.

Dadas as limitações de hardware e software, nos anos 1970, os analistas só conseguiram estudar alguns tipos de relacionamentos em populações não maiores que várias centenas. Além disso, não era possível obter listas completas dos membros da população e seus laços em muitas configurações, pois para tal são necessários milhares de dados a se computar. Os estudos clássicos que antecedem a década de 70 quando sociólogos urbanos ignoravam amizades fora do bairro e a inexistência de métodos que pudessem expressar a rede na sua íntegra, levava a resultados que podiam não apresentar a realidade da rede ao reduzir a análise ao foco egocêntrico (WELLMAN, 1997). Com as limitações, muitos analistas estruturais se concentraram no estudo de pequenas redes egocêntricas (redes pessoais) definidas do ponto de vista de indivíduos focais, no entanto, era impossível evitar o reducionismo metodológico.

Com o surgimento dos métodos analíticos em ARS (análise posicional, atributicional, decisional e interacional) e a aplicabilidade computacional para análise de dados em grandes escalas foi possível quantificar resultados a partir da perspectiva analítica a ser tomada. A análise computacional vem gradativamente sendo incorporada como ferramenta analítica em diversos campos de novos estudos em ARS. Silva e Silva (2016) afirmam que pesquisadores nos campos das ciências sociais e humanas têm a franca possibilidade de analisar aspectos de sua documentação de 
pesquisa por meio de recursos de cálculos sistemáticos, minimizando sobremaneira inferências, ou ilações, subjetivas.

A análise de redes, ou mais precisamente, os cálculos de rede, possibilitam a percepção de tendências de significados em grandes quantidades de documentos ou falas e reduzem substancialmente a necessidade de intervenção manual pelo pesquisador, aumentando a produtividade e sua capacidade de fazer mudanças relativas à direção que se observar, quando a dinâmica da pesquisa o exige. Tais procedimentos admitem ao pesquisador posicionar suas escolhas qualitativas baseadas na centralidade dos sentidos observáveis, aumentando significativamente a capacidade de interpretar a realidade social.

Os estudos em análise semântica desenvolvidos por Silva e Silva (2016) e Silva e Pereira (2016) buscam superar uma posição baseada na dualidade sujeito-objeto e apresentar a natureza relacional do conhecimento produzido na interação entre pesquisadores e objeto pesquisado, entrevistadores e entrevistados, onde a produção de sentidos não se fixa exclusivamente nos resultados dados nos softwares para análise, mas agrega procedimentos metodológicos para compor e organizar o sentido conferido aos dados pelos agentes produtores. Do contrário, os resultados estariam condicionados à bibliometria clássica, limitando a liberdade do pesquisador em explorar novos procedimentos.

A representação gráfica das relações entre as palavras necessita um trabalho precedente de preparação semântica antes que os dados sejam importados para um programa de análise de redes. A fase preliminar dos trabalhos implica na criação de uma camada de dados sobre o texto, na qual a intervenção do pesquisador é fundamental, todavia controlada e transparente ao leitor. Trabalhar com um software facilita o trabalho de análise e reanálise de dados.

Após o tratamento semântico os dados estão prontos para serem computados. Silva e Silva (2016), utilizaram o programa Gephi para a identificação de categorias através do cruzamento dos dados bibliométricos e semânticos. As categorias são o resultado computado e servem de orientação no uso dos termos centrais e periféricos das palavras. Ao mesmo tempo em que trazem as palavras mais usadas para o centro do grafo, aproximam por gravidade os termos mais próximos das categorias elencadas segundo o grau dado no programa computacional.

Com o objetivo de alcançar uma estrutura conceitual pertinente caracterizada por sua flexibilidade, plasticidade e eficácia, Arencibia-Jorge, Véga e Martí (2007) também aplicam conceitos da bibliometria e da análise semântica na substituição e recuperação de informações sobre prostática benigna hiperplasia (BPH) em um sistema de informação como metodologia de diagnóstico, acompanhamento e tratamento da patologia. Mais precisamente, o trabalho dos autores faz uso da bibliometria, da análise semântica e da ARS e percebe que as co-palavras que estruturam 
o corpus analisado não só permitem a observação da especificidade e uniformidade do significado das palavras atribuídas pelos autores (capital social), mas também a construção de termos representativos do domínio a que se vinculam.

Gama e Carvalho (2017) acreditam que enquanto o conceito de campo científico expressa o espaço das ações e trocas relacionadas a conformação das práticas científicas, o conceito de capital social refere-se a energia mobilizada por um ou mais atores na repercussão das pesquisas no campo acadêmico ao permitir a ampliação da disseminação da produção científica em um campo específico.

Para identificar os atores com maior índice de centralidade e fornecer apontamentos para a democratização do conhecimento sobre o tema da literatura científica nas instituições de ensino e pesquisa no Brasil, Gama e Carvalho (2017) utilizam da plataforma Web off Science e aplicam o método relacional de análise informacional (MRAI) na intenção de mapear a rede de coautoria científica de pesquisadores brasileiros sobre a temática repositórios digitais. Atualmente a base abrange cerca de 12.000 periódicos, além do conteúdo de cinco coleções: Science Citation Index Expanded (SCI-Expanded), Social Sciences Citation Index (SSCI), Arts \& Humanities Citation Index (A\&HCI), Conference Proceedings Citation Index-Science (CPCI-s) e Conference Proceeding Citation Index - Social Science \& Humanities (CPCI-ssh). Para a obtenção dos dados bibliométricos, assim como os grafos de redes apresentados no estudo as autoras utilizaram-se do software Ucinet 5.0, a fim de calcular a "centrality degree", que pode ser definida como o número de laços que incidem sobre um nó e representa o volume de capital social mobilizado na estrutura científica.

Conforme os resultados apresentados pelas autoras, nem sempre um autor que tem muitas publicações fará parte de uma grande rede de citações. O fato de um autor ser mais citado não implica necessariamente a ideia de ele ter mais impacto na rede. Isso pode ser explicado pelo conceito de aberturas estruturais (GAMA; CARVALHO, 2017), ou seja, um ator que na rede se relacionou em diversas relações, maximiza os seus contatos, o que emite a ele uma centralidade maior do que os demais.

Mesmo com toda a aplicação computacional, é relevante recordar que o uso de ferramentas quantitativas não substitui a leitura e a interpretação qualitativa sobre como as palavras dos textos formam significados, tal qual aponta Clemente (2015). Ao aplicar a análise bibliométrica e semântica para identificar as principais abordagens conceituais que dão sentidos diversos ao horizonte de expectativas do conceito da cidadania, o autor busca através da análise de conteúdo demonstrar que o interesse da decodificação recai não na mera descrição dos conteúdos, mas no que as nuances da linguagem pode ensinar. Destaca as características das obras centrais presentes nas 
conceituações sobre cidadania e apresenta seus particularismos. Com conceitos voltados para os horizontes de suas próprias sociedades, neste caso, alerta quanto a inclusão de um grande embasamento teórico referencial que denota a polissemia conceitual e o quanto os autores também são atores e buscam responder e influenciar seus próprios contextos históricos.

É com esta base teórico-conceitual que realizamos nosso trabalho de exploração de publicações anglófonas na área da ciência política para identificar as principais comunidades temáticas que utilizam a análise de redes como metodologia de trabalho e que, tendencialmente, devem compor os referenciais de estudos nesta área do conhecimento. Apresentamos a seguir os materiais e métodos utilizados na pesquisa.

\section{Materiais e métodos}

O trabalho de pesquisa foi realizado em quatro fases, a saber: a revisão bibliográfica e a coleta e tratamento dos dados; preparação e processamento em software para a análise bibliométrica, semântica e de redes; e a análise dos resultados propriamente dita. A busca inicial para coleta dos títulos e metadados dos artigos publicados em língua inglesa na área de ciência política foi realizada utilizando-se os recursos de busca avançada do Google Acadêmico ${ }^{4}$. Utilizamos o recurso de métricas do referido buscador ${ }^{5}$ para identificar as publicações com maior impacto na subcategoria political science da categoria political sciences ${ }^{6}$. Através deste recurso foram selecionados vinte periódicos com maior impacto de acordo com sua posição no índice h $5^{7}$ (de Hirsch) na subcategoria indicada. Tal classificação foi determinante como critério de seleção das produções centrais a serem consideradas para a coleta. O índice-h5 (do Google) é calculado usando apenas os artigos publicados nos últimos cinco anos completos recentes. Importante observar que todos os 20 periódicos selecionados apresentam a classificação máxima (A1) e circulação internacional no Qualis periódicos, disponível na Plataforma Sucupira ${ }^{8}$ do portal da Coordenação de Aperfeiçoamento de Pessoal de Nível Superior (CAPES). A Figura 01 traz uma lista dos vinte periódicos consultados para o presente trabalho.

\footnotetext{
$4 \quad$ https://scholar.google.com.br/

5 https://scholar.google.com.br/citations?view_op=metrics_intro\&hl=pt-BR

6 https://scholar.google.com.br/citations?view op=top venues\&hl=pt-BR\&vq=soc politicalscience

7 Sobre o índice H ver a publicação "Uso do Fator de impacto e do índice H para avaliar pesquisadores e publicações" (TOMAZ, ASSAD; MOREIRA, 2011).

8 https://sucupira.capes.gov.br/sucupira/
} 


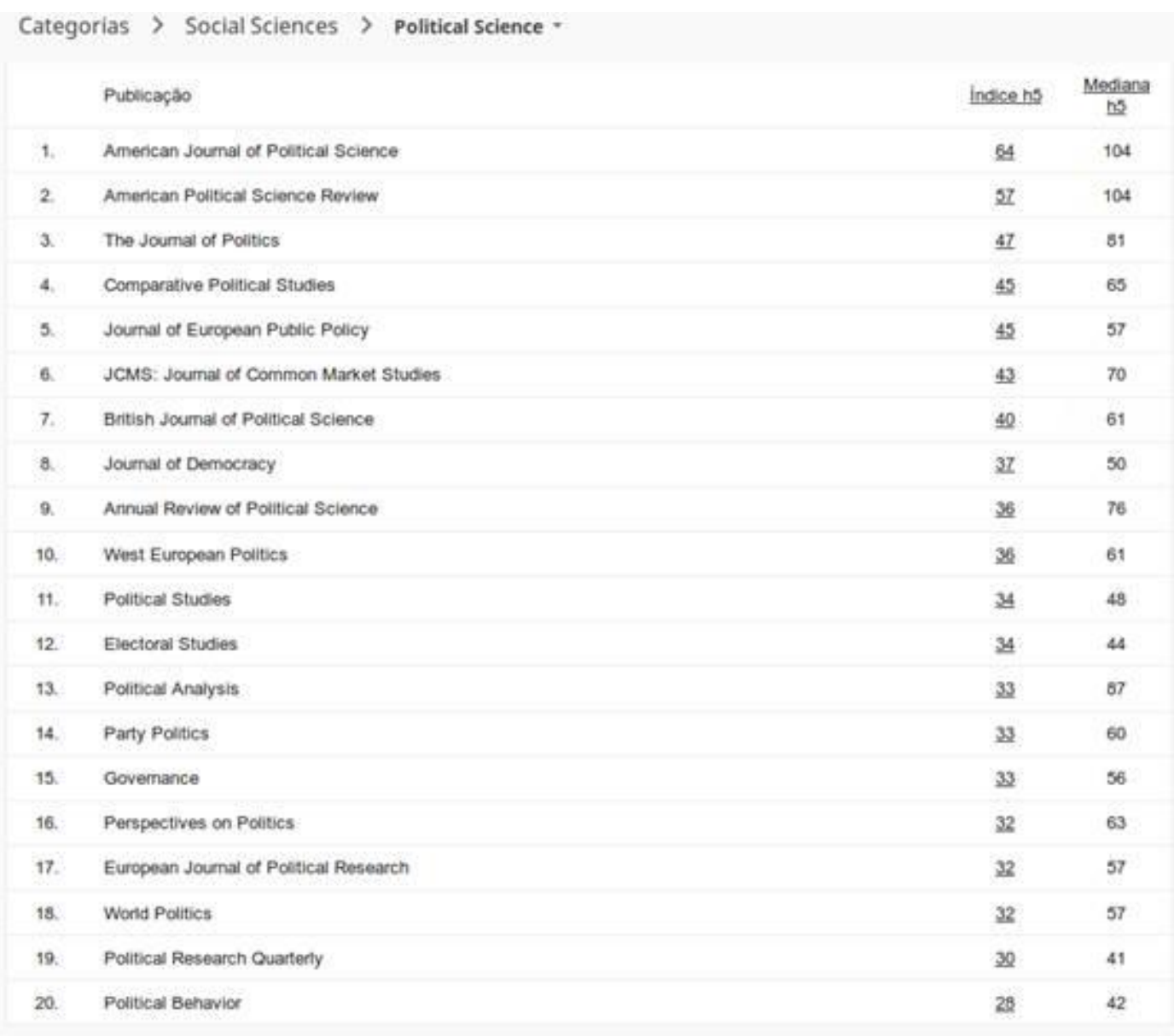

FIGURA 01: PERIÓDICOS SELECIONADOS PARA ESTUDO

FONTE: Google Acadêmico, 2017. Elaboração própria.

A busca por trabalhos que tenham utilizado a análise de redes como metodologia de estudo, em cada uma das 20 publicações listadas anteriormente, foi realizada utilizando como critério a expressão "social network analysis" em qualquer lugar do artigo. Cada publicação retornou um número variável de artigos correspondentes ao critério de busca, tendo sido totalizado uma coleta inicial de 211 títulos cuja listagem encontra-se disponível em https://goo.gl/k2vQcU. Os metadados relativos aos artigos selecionados foram obtidos mediante dois procedimentos: primeiramente todos os artigos foram salvos no dispositivo "minha biblioteca" (do próprio Google Acadêmico) e em seguida os registros foram exportados no padrão "endnote" para serem lidos pelo software gerenciador de referências Zotero. Do Zotero, as referências foram exportadas para serem lidas em planilha, no padrão "csv".

O passo seguinte foi acessar cada um dos artigos para obter, além do texto integral, o abstract, as palavras-chave e a conclusão. Uma dificuldade se deu porque grande parte da bibliografia utilizada é de acesso restrito e necessita de nome de usuário e senha para acesso integral. Geralmente, os artigos mais bem conceituados em língua inglesa (h5) tem restrições e 
somente o resumo está disponível abertamente, sendo costumeiramente comercializados. Para resolver esta dificuldade os periódicos foram acessados a partir de terminal fixo no Setor Litoral da Universidade Federal do Paraná (UFPR Litoral), com IP autenticado no servidor pela academia (IP) e a partir de acesso remoto identificado pela Comunidade Acadêmica Fada (CAFE) - (shibboleth), serviço provido pela Rede Nacional de Ensino e Pesquisa (RNP). Após acessar os documentos foi necessário extrair o abstract, keywords e as conclusions para inserir na planilha inicial que serviria para alimentar o programa para a análise de redes. $\mathrm{O}$ acesso e leitura preliminar dos textos resultou no expurgo de sete artigos que apenas citavam a análise de redes sem, contudo, assumi-la como metodologia de trabalho. Portanto, restaram 204 títulos considerados como "N" da coleta para a presente pesquisa. O conjunto de dados dos artigos selecionados está disponível em https://goo.gl/jXnXC4. Este arquivo contém informações das palavras-chave utilizadas na pesquisa, os autores abordados, o abstract de cada artigo, os periódicos pesquisados, as considerações de cada autor, uma classificação de cada artigo (periférico, aplicada, teórica), diante da temática análise de redes sociais.

Para que fosse possível construir a rede entre os artigos analisados buscamos homogeneizar as palavras-chave para reduzir a probabilidade de apontar resultados mascarados. Concordamos com Ruru e Lia (2017), quando afirmam que ferramentas de mineração de texto, devem ser exploradas com cautela para melhorar a precisão e eficiência da análise bibliométrica. Utilizamos um método de construção de redes semânticas que segue os seguintes critérios: (1) eliminação das palavras sem significados intrínsecos e (2) alteração das palavras restantes para sua forma canônica (FADIGAS et al., 2009, p. 169). Aplicamos então filtros para remoção de stop words e construção de relações binárias entre as palavras e/ou termos utilizados na produção de sentido, com a padronização destes termos para alimentação de colunas "Autor(es)" e "Palavraschave", em padrão de redes, com nós e arestas, que possibilitasse a leitura dos relacionamentos entre autor(es) e palavras-chave pelo programa Gephi (https://gephi.org/) de análise de redes sociais.

A tabela de nós consiste em tornar unívoco cada um dos valores (no nosso caso termos na forma de autores e palavras-chave) e a tabela de arestas consiste em informar para o programa cada um dos relacionamentos existentes entre autores e coautores e cada uma das palavras-chaves utilizadas em cada um dos textos. A planilha geral ficou composta por 783 linhas, ou seja, nossa rede é composta portanto por 783 nós, sendo eles 323 autores e coautores e 460 palavras-chave. A planilha de arestas contém 1.874 relacionamentos, ou seja, os 783 autores e palavras-chave estabelecem 1.874 conexões entre si. 
No Gephi, foram executados cálculos de rede que permitiram separar a grande rede em 18 componentes conectados. Sobre este grande componente conectado foram executados cálculos de modularidade para identificação das comunidades temáticas (autores e palavras-chave). Ambas as planilhas podem ser observadas nas Figuras 02 e 03 a seguir:

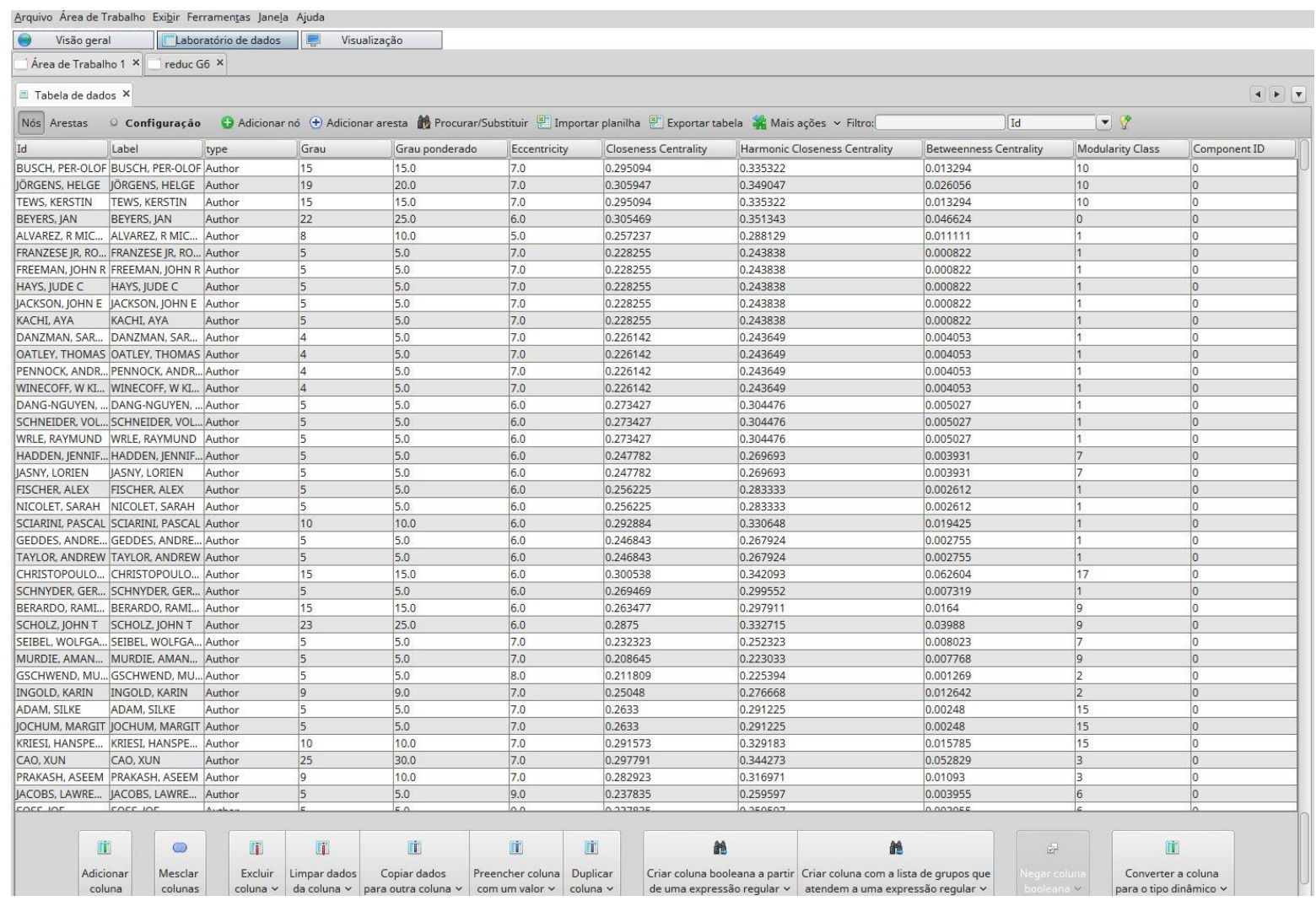

FIGURA 02: NÓS (PARCIAIS) COM CÁLCULOS

FONTE: programa Gephi. Elaboração própria. 


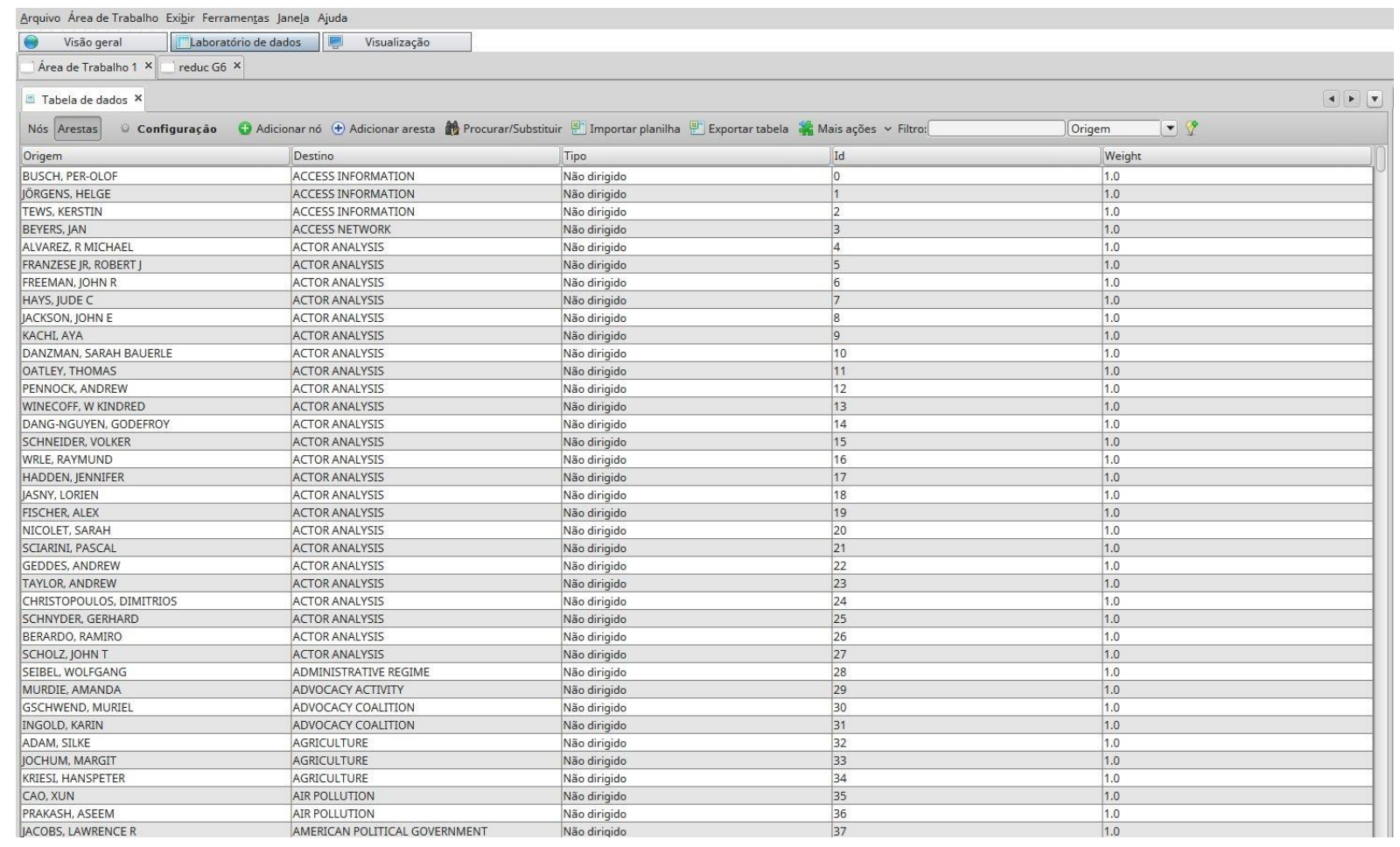

\section{FIGURA 03: ARESTAS (PARCIAL) COM CÁLCULOS}

FONTE: programa Gephi. Elaboração própria.

Além dos cálculos, o programa Gephi gera grafos de rede ilustrativos que serão exibidos a seguir ao longo da exploração e discussão dos resultados.

\section{As comunidades temáticas como método de exploração e estudos de uma área do conhecimento}

O grafo de nossa rede, resultante da conjugação dos 783 nós e 1.874 relacionamentos, ou arestas, pode ser observado na Figura 04, onde os autores estão representados pelos pontos azuis e as palavras-chave por pontos vermelhos e os relacionamentos pelas linhas de cor cinza. Os nós, ou pontos, estão graduados conforme a centralidade de grau apurada, ou seja, pela quantidade de relacionamentos simples que acumulam. Os rótulos correspondentes aos autores, coautores e palavras-chave foram suprimidos. 


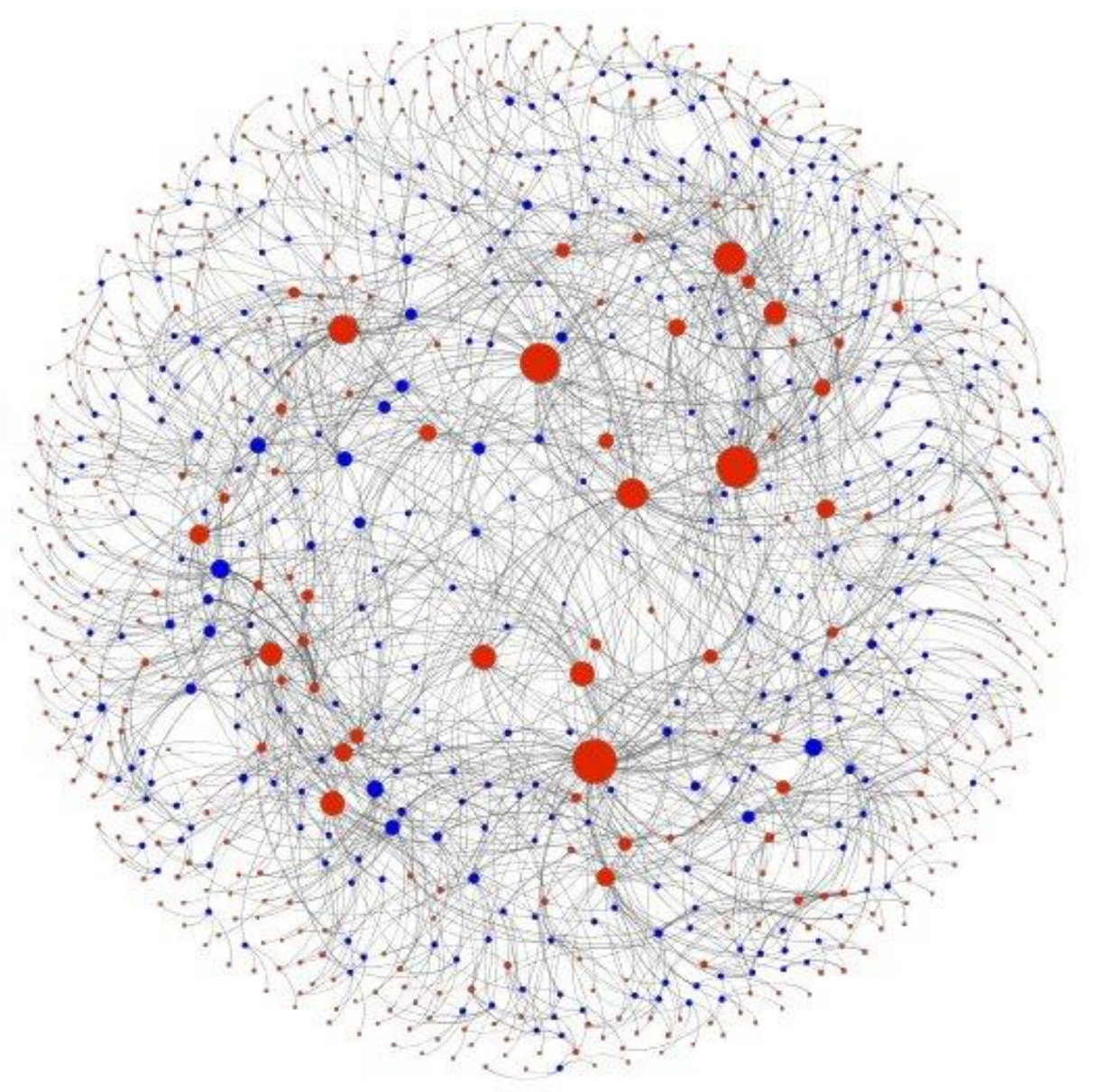

FIGURA 04: GRAFO DA REDE GERAL DE AUTORES E PALAVRAS-CHAVE FONTE: Elaboração própria.

Note-se que a primeira leitura que o grafo possibilita é de um conjunto reduzido de autores e palavras-chave que constituem a malha estruturante, ou central, da rede temática, enquanto podemos constatar também, na condição complementar, muitos pontos vermelhos periféricos, correspondentes a palavras-chave pouco citadas ou termos pouco compartilhados entre os autores dos artigos selecionados.

Sobre a rede geral representada anteriormente aplicamos cálculos de rede e obtemos como resultado a hierarquia dos nós, através dos cálculos de centralidade, e também um conjunto de dezoito partições não hierárquicas, ao que denominamos de comunidades, com o objetivo de verificar se existem e quais são os agrupamentos temáticos formadas no interior da rede em função da proximidade entre autores e palavras-chave. A distribuição por comunidades revela a tessitura de sub-redes, ou sub-grupos temáticos, de acordo com a proximidade entre os nós (autores e palavraschave). Uma comunidade é o resultado da análise qualitativa das relações, cujo critério é a 
proximidade que é estabelecida na resolução utilizada ${ }^{9}$. Na resolução 1.0 foi possível identificar dezoito comunidades que podem ser observadas na Figura 05, onde cada comunidade está representada por uma cor específica.

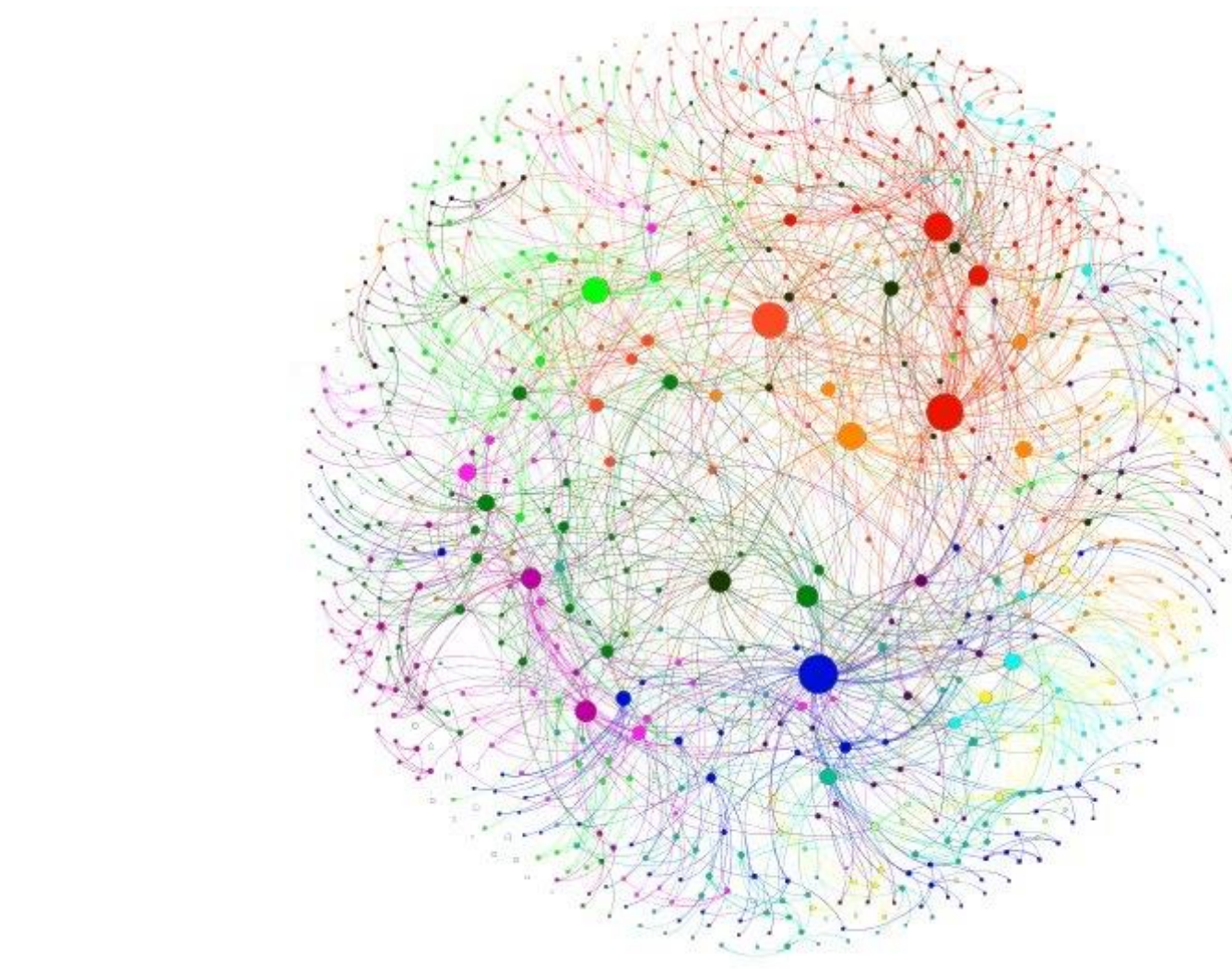

FIGURA 05: GRAFO DAS 18 COMUNIDADES TEMÁTICAS DA REDE GERAL DE AUTORES E PALAVRASCHAVE

FONTE: Elaboração própria.

O grafo apresentado na figura anterior pode também ser apresentado separando-se cada uma das comunidades, utilizando para isto a distribuição por eixos radiais, desta forma é possível obter uma visão bastante clara das comunidades, as quais podem ser observadas na Figura 06.

9 O algoritmo de modulação e constituição das comunidades podem ser consultados em Blondel et al. (2008) ou Lambiotte et al. (2008). 


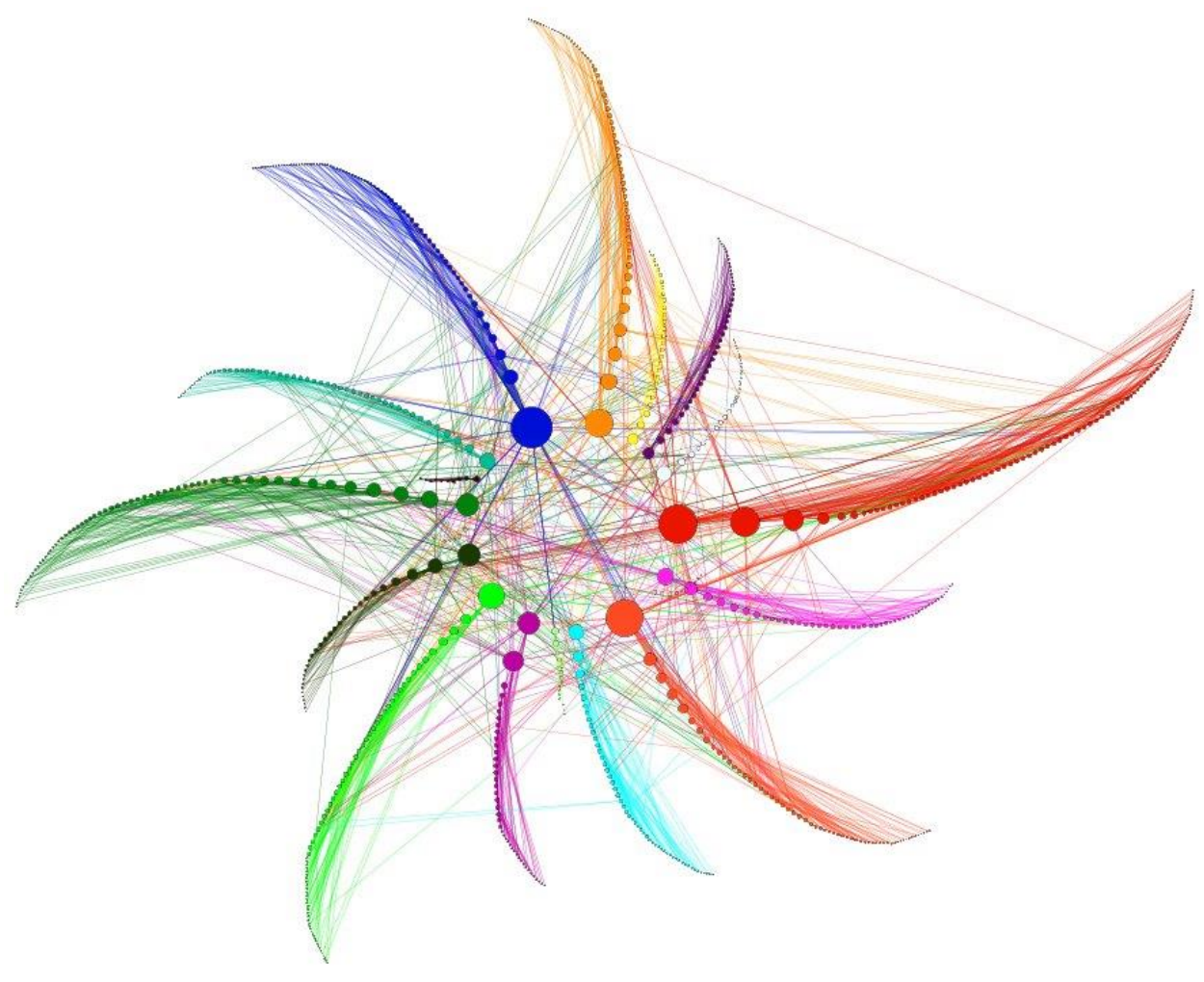

FIGURA 06: GRAFO DAS 18 COMUNIDADES TEMÁTICAS DA REDE GERAL DE AUTORES E PALAVRASCHAVE - DISTRIBUIÇÃO POR EIXO RADIAL

FONTE: Elaboração própria.

O intenso (intra) relacionamento entre os autores e palavras-chave de cada um dos dezoito módulos, expressos nas cores do grafo anterior contrasta com o reduzido inter-relacionamento entre os respectivos módulos. Tal dinâmica confere maior relevância aos resultados pois as comunidades estão bastante bem definidas. Importante reafirmar que a formação de uma comunidade temática é resultante de uma análise qualitativa das relações cujo critério é o conjunto de proximidades estabelecidas por cada nó (palavra-chave e autor) na rede. O algoritmo e a modularidade utilizados para a constituição das comunidades podem ser consultados em Blondel et al. (2008) e Lambiotte et al. (2008). Na tabela 01 estão listadas as dezoito comunidades estudadas (0-17): 
TABELA 01 COMUNIDADES TEMÁTICAS EM ARS

\begin{tabular}{|c|c|c|}
\hline COMUNIDADE & $\mathbf{N}$ autores & $\mathrm{N}$ palavras-chave \\
\hline 0 EUROPEAN UNION & 25 & 51 \\
\hline 1 ACTOR ANALYSIS & 25 & 28 \\
\hline 2 REGULATORY AGENCY & 04 & 07 \\
\hline 3 GOVERNMENT & 25 & 36 \\
\hline 4 CHOICE BEAWIOR & 02 & 04 \\
\hline 5 POLITICAL NETWORK & 14 & 17 \\
\hline 6 POLITICAL SCIENCE & 30 & 45 \\
\hline 7 NETWORK ANALYSIS & 18 & 25 \\
\hline 8 EMIGRATION & 05 & 11 \\
\hline 9 POLITICAL BEAWIOR & 17 & 35 \\
\hline 10 SOCIAL NETWORK ANALYSIS & 21 & 38 \\
\hline 11 POLITICAL PARTICIPACION & 03 & 07 \\
\hline 12 INTERNATIONAL RELATIONS & 20 & 27 \\
\hline 13 POLITICAL PARTY & 44 & 39 \\
\hline 14 DEMOCRACY & 11 & 20 \\
\hline 15 ELITE & 18 & 23 \\
\hline 16 IMMIGRATION & 14 & 14 \\
\hline 17 SOCIAL NETWORK & 28 & 33 \\
\hline
\end{tabular}

FONTE: Elaboração própria.

Após a identificação destas dezoito comunidades exportamos os dados gerados no Gephi, relativos a cada uma delas, e procedemos então a uma análise e discussão das principais comunidades temáticas. Importa ainda registrar que para efeitos de análise da rede o recurso utilizado foi primordialmente as tabelas de cálculos, através das quais podemos "ver" e "ler" as redes. Os grafos de rede funcionam, portanto, pela visualização, como um elemento auxiliar para a compreensão dos relacionamentos analisados nas tabelas.

Utilizando como critério o número de autores da base, o número de conexões, a quantidade de artigos, a média de autores por artigo e a média de coautorias identificadas (MUCHERONI; FUNARO, 2015) apresentamos a seguir o agregado das seis comunidades temáticas selecionadas 
(political party, political science, european union, government, actor analysis e international relations) e o detalhamento da primeira delas.

As seis comunidades agregadas são constituídas por 395 nós, sendo 170 autores e 225 palavras-chave, que estabelecem um total de 801 relacionamentos entre si. O grafo resultante desta agregação pode ser observado na Figura 07 a seguir.

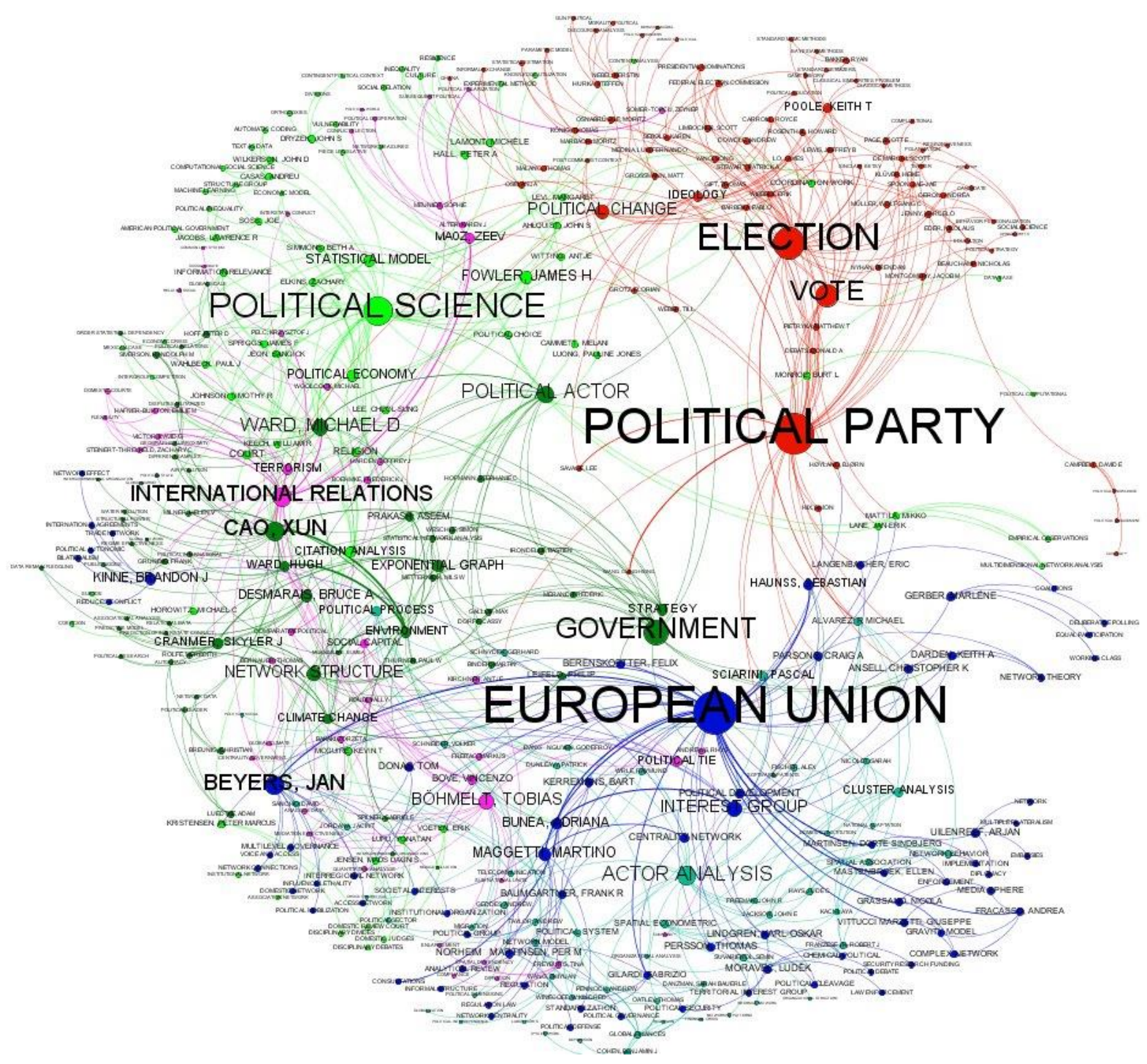

FIGURA 07: GRAFO DAS SEIS PRINCIPAIS COMUNIDADES TEMÁTICAS

FONTE: Elaboração própria.

O grafo anterior evidencia o volume e a dimensão que as principais comunidades temáticas alcançam em relação ao conjunto da rede. Para uma melhor síntese perceptiva deste conjunto, o Quadro 01 a seguir relaciona o principal autor, e suas obras, em cada uma dessas seis comunidades temáticas identificadas no campo da ciência política entre os anos de 2011 e 2016. 
QUADRO 01: AUTORES E ARTIGOS CENTRAIS NAS PRINCIPAIS COMUNIDADES TEMÁTICAS

\begin{tabular}{|c|c|c|}
\hline $\begin{array}{l}\text { COMUNIDADE } \\
\text { TEMÁTICA }\end{array}$ & AUTOR (ES) & OBRA / PUBLICAÇÃO \\
\hline \multirow[b]{2}{*}{ Political Party } & BAKKER, R.; POOLE, K. T. & $\begin{array}{l}\text { Bayesian metric multidimensional scaling. Political } \\
\text { Analysis, v. } 21, \text { n. 1, p. } 125-140,2013 .\end{array}$ \\
\hline & $\begin{array}{c}\text { CARROLL, Royce; LEWIS, } \\
\text { Jeffrey B.; LO, James; } \\
\text { POOLE, Keith T.; } \\
\text { ROSENTHAL, Howard. } \\
\end{array}$ & $\begin{array}{l}\text { The structure of utility in spatial models of } \\
\text { voting.American Journal of Political Science, v. 57, n. 4, p. } \\
\text { 1008-1028, } 2013 \text {. }\end{array}$ \\
\hline \multirow{2}{*}{ Political Science } & $\begin{array}{l}\text { FOWLER, J. H.; JOHNSON, } \\
\text { T. R.; SPRIGGS, J. F.; JEON, } \\
\quad \text { S.; WAHLBECK, P. J. }\end{array}$ & $\begin{array}{l}\text { Network analysis and the law Measuring the legal } \\
\text { importance of precedents at the US Supreme Court. } \\
\text { Political Analysis, p. 324-346, } 2007 \text {. }\end{array}$ \\
\hline & FOWLER, J. H. & $\begin{array}{l}\text { Connecting the Congress: a study of cosponsorship } \\
\text { networks. Political Analysis, p. 456-487, } 2006 .\end{array}$ \\
\hline \multirow{3}{*}{ European Union } & \multirow{2}{*}{ BEYERS, J. } & $\begin{array}{l}\text { Gaining and seeking access The European adaptation of } \\
\text { domestic interest associations. European Journal of } \\
\text { Political Research, v. 41, n. 5, p. 585-612, } 2002 \text {. }\end{array}$ \\
\hline & & $\begin{array}{l}\text { Voice and access Political practices of European interest } \\
\text { associations. European Union Politics, v. 5, n. 2, p. 211- } \\
\text { 240, } 2004 \text {. }\end{array}$ \\
\hline & BEYERS, J.; DONAS, T. & $\begin{array}{c}\text { Inter-regional networks in Brussels Analyzing the } \\
\text { information exchanges among regional offices. European } \\
\text { Union Politics, p. } 1465116514536269,2014 .\end{array}$ \\
\hline \multirow{2}{*}{ Government } & $\begin{array}{l}\text { WARD, H.; CAO, X.; } \\
\text { MUKHERJEE, B. }\end{array}$ & $\begin{array}{l}\text { State capacity and the environmental investment gap in } \\
\text { authoritarian states. Comparative Political Studies, v. 47, n. } \\
\text { 3, p. 309-343, } 2014 \text {. }\end{array}$ \\
\hline & $\begin{array}{l}\text { WARD, M. D.; SIVERSON, } \\
\text { R. M.; CAO, X. }\end{array}$ & $\begin{array}{l}\text { Disputes, democracies, and dependencies: a reexamination } \\
\text { of the Kantian peace. American Journal of Political } \\
\text { Science, v. 51, n. 3, p. 583-601, } 2007 .\end{array}$ \\
\hline Actor Analysis & SCIARINI, P. & $\begin{array}{l}\text { Eppure si muove the changing nature of the Swiss } \\
\text { consensus democracy. Journal of European Public Policy, } \\
\text { v. } 21 \text {, n. } 1 \text {, p. } 116-132,2014 .\end{array}$ \\
\hline $\begin{array}{l}\text { International } \\
\text { Relation }\end{array}$ & BOVE, V.; BÖHMELT, T. & $\begin{array}{c}\text { Does immigration induce terrorism The Journal of Politics, } \\
\text { v. } 78, \text { n. } 2 \text {, p. } 572-588,2016 .\end{array}$ \\
\hline
\end{tabular}

FONTE: Elaboração própria.

A identificação das comunidades temáticas realizada e apresentada em tabelas, grafos e quadros permite, por exemplo, orientar uma linha de pesquisa em destaque nas publicações anglófonas no campo da ciência política. No caso em análise, pode-se orientar os pesquisadores do campo da ciência política que utilizam, ou têm interesse em utilizar, a ARS em suas respectivas agendas de investigação. A exploração focada em uma comunidade, ou ainda de cada uma das comunidades, permite demonstrar que o método proposto é capaz de proporcionar ao pesquisador a 
identificação de quais obras são centrais em determinada temática e, consequentemente, se impõem para a leitura, seja ela crítica ou corroborativa das ideias e teses expressas por seus autores.

A comunidade temática political party é composta por 44 autores e coautores e trinta e nove palavras-chave que estabelecem 457 relacionamentos na rede, dos quais 161 correspondem especificamente a esta comunidade e podem ser observados no grafo da figura 08. Os nós estão dimensionados de acordo com o grau, ou seja, do número de relacionamentos estabelecidos na rede.

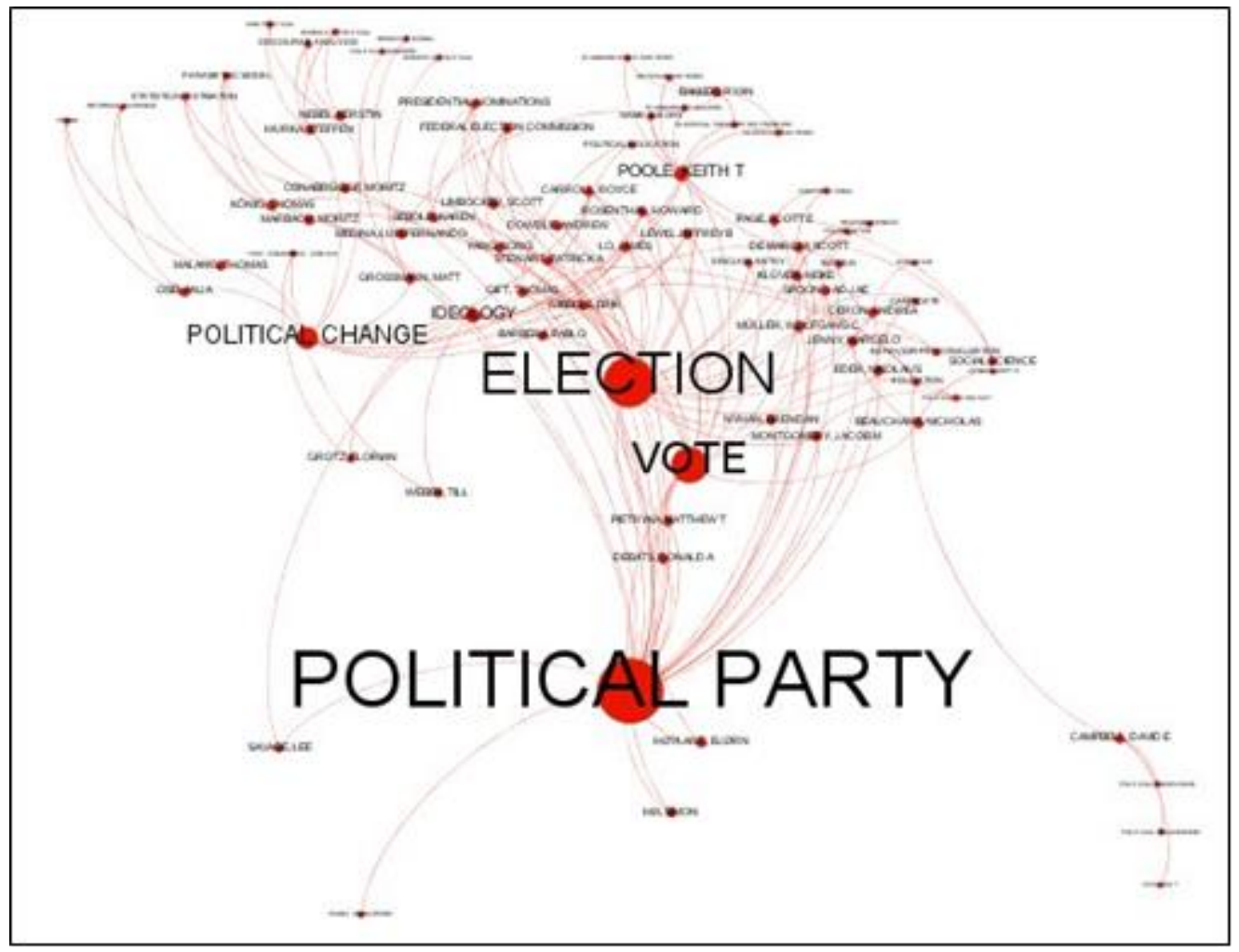

FIGURA 08: GRAFO DA COMUNIDADE POLITICAL PARTY

FONTE: Elaboração própria.

$\mathrm{Na}$ figura anterior, em destaque podemos observar os termos election e vote, os quais, compõem, junto com political party, os termos centrais, ou as palavras-chave mais expressivas desta comunidade, seguidas de political change e ideology. Essa comunidade tem como principais autores Ryan Bakker e Keith Poole. A Tabela 02 apresenta os dez primeiros termos (palavra-chave ou autor/coautor) por ordem de grau (degree) que aparecem na figura anterior. 
TABELA 02: PALAVRAS-CHAVE E AUTORES NA COMUNIDADE POLITICAL PARTY

\begin{tabular}{lcc}
\hline \multicolumn{1}{c}{ Label } & Type & Degree \\
\hline POLITICAL PARTY & Palav_Chv & 60 \\
\hline ELECTION & Palav_Chv & 45 \\
\hline VOTE & Palav_Chv & 31 \\
\hline POLITICAL CHANGE & Palav_Chv & 17 \\
\hline POOLE, KEITH T & Author & 10 \\
\hline IDEOLOGY & Palav_Chv & 10 \\
\hline BAKKER, RYAN & Author & 5 \\
\hline MEDINA, LUIS FERNANDO & Author & 5 \\
\hline EDER, NIKOLAUS & Author & 5 \\
\hline JENNY, MARCELO & Author & 5 \\
\hline
\end{tabular}

FONTE: Elaboração própria.

No Quadro 02 que segue é possível observar os trabalhos referentes aos autores mais significativos desta comunidade. Apresentamos neste quadro a bibliografia indicada para pesquisadores que se dediquem a comunidade political party.

QUADRO 02: PRINCIPAIS AUTORES E OBRAS NA COMUNIDADE POLITICAL PARTY

\begin{tabular}{|c|c|}
\hline AUTOR(ES) & OBRA / PUBLICAÇÃO \\
\hline BAKKER, R.; POOLE, K. T. & $\begin{array}{c}\text { Bayesian metric multidimensional scaling. Political } \\
\text { Analysis, v. 21, n. 1, p. 125-140, 2013. }\end{array}$ \\
\hline $\begin{array}{c}\text { CARROLL, Royce; LEWIS, Jeffrey B.; LO, } \\
\text { James; POOLE, Keith T.; ROSENTHAL, Howard. }\end{array}$ & $\begin{array}{c}\text { The structure of utility in spatial models of voting. American } \\
\text { Journal of Political Science, v. 57, n. 4, p. 1008-1028, 2013. }\end{array}$ \\
\hline EDER, N.; JENNY, M.; MÜLLER, W. C. & $\begin{array}{c}\text { Winning over voters or fighting party comrades Personalized } \\
\text { constituency campaigning in Austria. Electoral Studies, v. } \\
\text { 39, p. 316-328, 2015. }\end{array}$ \\
\hline MEDINA, L. F. & $\begin{array}{c}\text { The analytical foundations of collective action theory a } \\
\text { survey of some recent developments. Annual Review of } \\
\text { Political Science, v. 16, p. 259-283, 2013. }\end{array}$ \\
\hline CONSIDINE, M.; LEWIS, J. M. & $\begin{array}{c}\text { Innovation and innovators inside government from } \\
\text { institutions to networks. Governance, v. 20, n. 4, p. 581-607, } \\
\text { 2007. }\end{array}$ \\
\hline CAMPBELL, D. E. & $\begin{array}{c}\text { Social networks and political participation. Annual Review } \\
\text { of Political Science, v. 16, p. 33-48, 2013. }\end{array}$ \\
\hline
\end{tabular}

FONTE: Elaboração própria.

Após mapeadas as comunidades temáticas e identificados os autores centrais em cada comunidade e suas respectivas obras, torna-se possível realizar uma leitura direcionada, seletiva, ao núcleo da produção em uma determinada área do conhecimento. A listagem completa com todos os 
textos que compõem as comunidades temáticas estão disponíveis para pesquisadores e estudiosos no endereço: https://goo.gl/cGJTJn.

O mesmo trabalho de discussão das principais obras identificadas em cada comunidade temática, a partir dos autores centrais e palavras-chaves, pode perfeitamente ser realizado nas demais comunidades que compõem a rede de publicações que utilizam análise de redes como um recurso de investigação em ciência política.

\section{Conclusão}

O conjunto de procedimentos utilizados para responder ao questionamento colocado para este trabalho (quais os temas e autores centrais que publicam em língua inglesa na área de ciência política abordando a análise de redes sociais?) gerou uma ferramenta generalizável com a qual é possível "atalhar" caminhos e identificar os principais autores a serem buscados e lidos em uma determinada área do conhecimento.

Ante o caráter inovador e o potencial de generalização do trabalho realizado, buscamos trazer a baila ferramentas científico metodológicas para apresentar um quadro das comunidades temáticas em ciência política nos anos de 2011 e 2016. Entendemos que muito foi feito e há muito a se fazer quanto ao aprofundar em cada comunidade abordada. Os resultados puderam comprovar a valia dos métodos em análise semântica e ARS para a seleção de um corpus. O "mapa" temático em categorias em ARS fornecido pode contribuir para pesquisas mais aprofundadas sob os temas indicados.

Finalmente, a partir do refinamento das metodologias de análise acreditamos ter alcançado os objetivos iniciais fazendo uso dos mais atuais mecanismos computacionais para análise, observação e identificação de comunidades temáticas em ciência política e análise de redes sociais.

\section{Referências}

ARENCIBIA-JORGE, R.; VEGA-ALMEIDA, R. L.; MARTÍ-LAHERA; Y. Domain analysis for the construction of a conceptual framework: a case study. LIBRES Library and Information Science Research Electronic Journal. Volume 17, Issue 2, p. 1-26, September 2007.

BAKKER, R.; POOLE, K. T. Bayesian metric multidimensional scaling. Political Analysis, v. 21, n. 1, p. 125-140, 2013. 
BERRY, F. S. et al. Three traditions of network research: What the public management research agenda can learn from other research communities. Public administration review, v. 64, n. 5, p. 539-552, 2004.

BEYERS, J. Gaining and seeking access The European adaptation of domestic interest associations. European Journal of Political Research, v. 41, n. 5, p. 585-612, 2002.

BEYERS, J. Voice and access Political practices of European interest associations. European Union Politics, v. 5, n. 2, p. 211-240, 2004.

BEYERS, J.; DONAS, T. Inter-regional networks in Brussels: Analyzing the information exchanges among regional offices. European Union Politics, p. 1465116514536269, 2014.

BLONDEL, V. D. et al. Fast unfolding of communities in large networks. Journal of statistical mechanics: theory and experiment, v. 2008, n. 10, p. P10008, 2008.

BORGATTI, S. et al. Network analysis in the social sciences. Science, v. 13; 323, p. 892-895.

BOVE, V.; BÖHMELT, T. Does immigration induce terrorism The Journal of Politics, v. 78, n. 2, p. 572-588, 2016.

BUNEA, A.; BAUMGARTNER, F. R. The state of the discipline: authorship, research designs, and citation patterns in studies of EU interest groups and lobbying. Journal of European Public Policy, v. 21, n. 10, p. 1412-1434, 2014.

CAMPBELL, D. E. Social networks and political participation. Annual Review of Political Science, v. 16, p. 33-48, 2013.

CARROLL, R.; LEWIS, J. B.; LO, J.; POOLE, K. T.; ROSENTHAL, H. The structure of utility in spatial models of voting. American Journal of Political Science, v. 57, n. 4, p. 1008-1028, 2013.

CLEMENTE, A. J. O Conceito de Cidadania nas Ciências Sociais Brasileira: uma análise a partir do Scielo (1989-2013). 2015, Universidade Federal do Rio Grande do Sul, Instituto de Filosofia e Ciências Humanas, TESE, Porto Alegre, 2015.

CONSIDINE, M.; LEWIS, J. M. Innovation and innovators: Inside Government From Institutions to Networks. Governance, v. 20, n. 4, p. 581-607, 2007.

EDER, N.; JENNY, M.; MÜLLER, W. C. Winning over voters or fighting party comrades? Personalized constituency campaigning in Austria. Electoral Studies, v. 39, p. 316-328, 2015.

FADIGAS, I. S. et al. Análise de redes semânticas baseada em títulos de artigos* de periódicos científicos: o caso dos periódicos de divulgação em educação matemática. Educação Matemática Pesquisa, v. 11, n. 1, p. 167-193, 2009.

FERREIRA, A. G. C. Bibliometria na avaliação de periódicos científicos. DataGramaZero, v. 11, n. 3, p. 1-9, 2010.

FOWLER, J. H. Connecting the Congress: a study of cosponsorship networks. Political Analysis, p. 456-487, 2006. 
FOWLER, J. H. et al. Network analysis and the law: Measuring the legal importance of precedents at the US Supreme Court. Political Analysis, p. 324-346, 2007.

GAMA, S. I.; CARVALHO, S. L. Tendências e Perspectivas de Pesquisa sobre Repositórios Digitais no Brasil: uma análise de rede sociais. Reciis. v.11, p. 1-14, 2017.

LAMBIOTTE, R.; DELVENNE, J.-C.; BARAHONA, M. Laplacian dynamics and multiscale modular structure in networks. Physics and Society, arXiv preprint arXiv:0812.1770, 2008.

LI, N.; HAN, R.; LU, X.. Bibliometric analysis of research trends on solid waste reuse and recycling during 1992-2016. Resources, Conservation and Recycling, v. 130, p. 109-117, 2018.

LIMA, Gonçalves, A. Uso de resumos e palavras-chave em Ciências Sociais: uma avaliação. Encontros Bibliométricos, v. 13, n. 26, p. 78-93, 2008.

MEDINA, L. F. The analytical foundations of collective action theory: A survey of some recent developments. Annual Review of Political Science, v. 16, p. 259-283, 2013.

MIZRUCHI, M. S. Análise de redes sociais: avanços recentes e controvérsias atuais. Revista de Administração de Empresas, v. 46, n. 3, p. 72-86, 2006.

MUCHERONI, M. L.; FUNARO, D. Análise de redes sociais na colaboração científica. XVI Encontro Nacional de Pesquisa em ciência da informação (XVI enancib), mimeo, USP, 2015.

SCOTT, J. Trend Report Social Network Analysis. Sociology, Vol. 22, No. 1 (February, 1988), pp. 109-127. Sage Publications, Ltd.

SILVA, E. A.; SILVA, J. M. Ofício, engenho e arte: inspiração e técnica na análise de dados qualitativos. Revista Latino-Americana de Geografia e Gênero, v. 7, n. 1, p. 132-154, 2016.

SILVA, E. A.; PEREIRA, M. A. M. Balanço aos 20: a Revista de História Regional no campo historiográfico brasileiro. Revista de História Regional, v. 21, n. 2, p. 307-337, 2016.

WARD, M. D.; SIVERSON, R. M.; CAO, X. Disputes, democracies, and dependencies: A reexamination of the Kantian peace. American Journal of Political Science, v. 51, n. 3, p. 583601, 2007.

WARD, H.; CAO, X.; MUKHERJEE, B. State capacity and the environmental investment gap in authoritarian states. Comparative Political Studies, v. 47, n. 3, p. 309-343, 2014.

WELLMAN, B. El análisis estructural de las redes sociales: del método y la metáfora a la teoría y la sustancia. Debates en sociología, n. 22, p. 47-97, 1997.

Artigo recebido em 19/01/2018. Aceito para publicação em 08/11/2018. 